\title{
Paratracheal Stripe Sign: The Chest X-ray Itself Voices Pathology
}

Bunkar Motilal ${ }^{1}$, Takhar Rajendra ${ }^{1,{ }^{*}}$, Arya Savita$^{2}$ and Mirdha Saroj ${ }^{3}$

${ }^{1}$ Department of Respiratory Medicine, Government Medical College, Kota, Rajasthan, India

${ }^{2}$ Government Medical College, Kota, Rajasthan, India

${ }^{3}$ Government Hospital, Nagaur, Rajasthan, India

\begin{abstract}
The chest X-ray (antero-posterior and lateral view) is the basic radiographic investigation to assess the chest abnormality, however, for better delineation of parenchymal and mediastinal diseases the role of computed tomography (CT) is also increasing. There are many diseases which give a peculiar and characteristic appearance (lines, stripes, and signs) on either conventional chest X-ray or CT. A radiologist and physician must be aware of these unusual appearances. Here, we are discussing such an important and common radiological sign, which is often missed - "Paratracheal stripe sign".
\end{abstract}

Keywords: Paratracheal stripe; Chest X ray; Radiological sign Introduction

Chest $\mathbf{X}$-ray is such a fundamental and basic diagnostic tool for pulmonary physicians as electrocardiogram for cardiologists. Chest $\mathbf{X}$-ray has unique lines, stripes or signs and their interpretations directly tell us about the disease process. These lines include anterior or posterior junction lines and stripes are the left and right paratracheal ones and the posterior tracheal stripe. Interpretation of chest radiographs necessitates radiologists as well as physicians to distinguish these important mediastinal lines and stripes. The inability to diagnose an abnormality on chest radiograph owing to the displacement of one of these lines or stripes may lead to failure to request a potentially valuable CT examination and causing a diagnostic dilemma. Here, we are discussing a classical radiological sign, suspected on conventional chest $\mathbf{X}$-ray and further investigated by CT chest.

\section{Case}

A 40-years old female came to outpatient department with complaints of dry cough since three months. She denied for chest pain, fever, hemoptysis, loss of appetite and/or weight. She had no history of smoking or tuberculosis. All routine blood investigations were within normal limit including human immunodeficiency virus serology except raised ESR (110 mm in the first hour). A skiagram chest posteroanterior (PA) view was done for further work-up (Figure 1).

Q.1. Describe the chest $\mathbf{X}$-ray and identify the radiological sign?

Q.2. What are the differential diagnoses?

\section{Answers}

Ans.1. Chest $\mathbf{X}$-ray PA view is showing a homogenous elongated opacity in the right paratracheal region that is extending from right hilum below to upward upto the clavicle with smooth margins. Sign is also termed as "Paratracheal strip sign".

Ans.2. The differential diagnosis comprises of space occupying lesions in the middle compartment of the upper mediastinum or superior mediastinum. These include Lymphadenopathy (tubercular, malignant), malignancy (thyroid, parathyroid, tracheal), thymic lesions (hyperplasia, thymoma, carcinoma, cyst and thymolipoma), lipoma, mediastinal fat, mediastinal hematoma, retrosternal goitre, pleural effusion $^{\star}$ and tracheal stenosis ${ }^{*}{ }^{*}$ very rarely) [1].

\section{Discussion}

The paratracheal strip sign is a normal finding on ski gram chest PA view, visible due to silhouette sign. When the visceral and

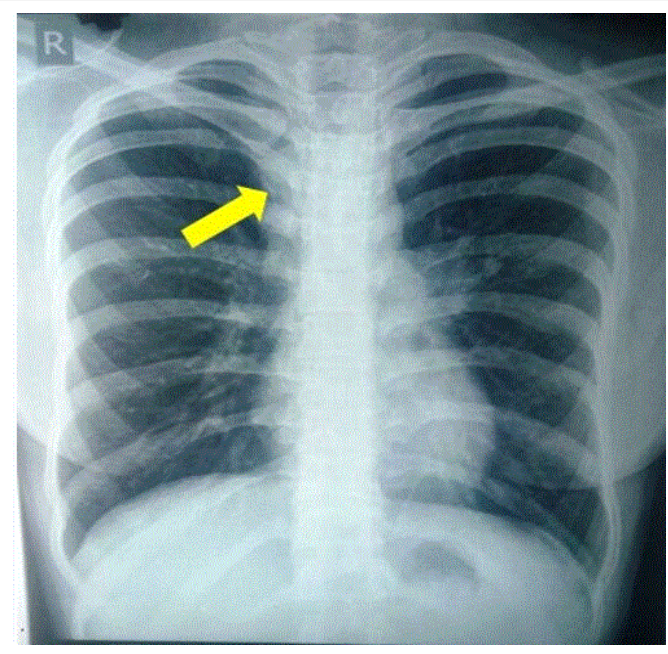

Figure 1: Chest $\mathrm{x}$-ray PA view showing a homogenous elongated opacity in the right paratracheal region extending below and upward to the clavicle with smooth margins.

parietal pleura of upper lobe come in contact with the lateral border of the trachea and the intervening mediastinal fat, air within the lung and trachea outlines this rare entity to form paratracheal stripe [2]. Normally, the size of stripe ranges from 1- $4 \mathrm{~mm}$. Any change in the stripe either thickness more than $4 \mathrm{~mm}$, any obliteration and distortion almost always suggestive of pathology.

It has two varieties:-

a.) Right paratracheal stripe sign: This one is the most commonly seen mediastinal line or stripe in skiagram chest approximate $90 \%$. It extends from the clavicle above to the right tracheobronchial angle at

*Corresponding author: Takhar Rajendra, MD, Assistant Professor, Department of Respiratory Medicine, Government Medical College, Kota, Rajasthan, India, Tel: +919784006021; E-mail: drrajtakhar@gmail.com

Received November 19, 2015; Accepted December 07, 2015; Published December 11, 2015

Citation: Motilal B, Rajendra T, Savita A, Saroj M (2015) Paratracheal Stripe Sign: The Chest X-ray Itself Voices Pathology. J Clin Respir Dis Care 1: 102. doi: 10.4172/ JCRDC.1000102

Copyright: ( 2015 Motilal B, et al. This is an open-access article distributed under the terms of the Creative Commons Attribution License, which permits unrestricted use, distribution, and reproduction in any medium, provided the original author and source are credited. 

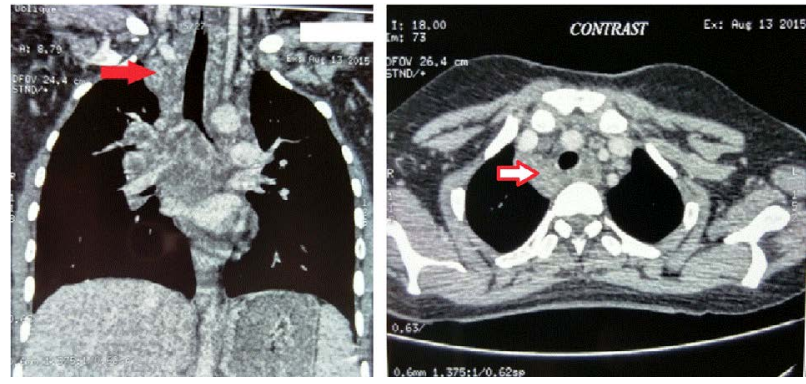

Figure 2: Contrast enhanced computed tomography showing heterogeneously enhancing multiple mediastinal (paratracheal) enlarged lymph nodes on right side (arrow).

the level of azygous arch and usually in continuity with right side great vessels superiorly [3].

b.) Left paratracheal strip sign: It extends from the aortic arch to the left subclavian artery. The left paratracheal stripe is seen less frequently than right one, as it may be obscured by contact between the left lung and the proximal left common carotid artery or left subclavian artery posteriorly [4].

Final diagnosis of our case was right side tubercular lymphadenopathy, that was confirmed by contrast enhanced computed tomography (CECT) scan of the chest (Figure 2), which revealed heterogeneously enhancing multiple paratracheal enlarged lymph nodes on right side with some areas of internal necrosis, followed by CT guided fine needle aspiration cytology from the lymph nodes. The capability of recognizing an abnormal chest radiograph on the basis of the displacement, deformation or obscuration of paratracheal stripe and apprehensive one of differential diagnosis is often mandatory prior to requesting a potentially useful CECT scan examination.

\section{Learning point}

1. Even with the increased dependence on CT in the assessment of chest disease, traditional frontal and lateral chest radiography remains a valuable tool in this setting.

2. The Radiologists, physicians and trainees must be accustomed with the anatomic basis of paratracheal stripes that are seen on chest skiagram and recognize the normal and abnormal appearances of the stripes to cultivate an appropriate differential diagnosis prior to obtaining additional information with CECT chest.

\section{References}

1. Marano R, Liguori C, Savino G, Merlino B, Natale L, et al. (2011) Cardiac silhouette findings and mediastinal lines and stripes: radiograph and CT scan correlation. Chest 139: 1186-1196.

2. h t t p : // w w w. a untminnie e u r o p e.com/index aspx?sec=ser\&sub=def\&pag=dis\&ltemID $=608038$.

3. Gibbs JM, Chandrasekhar CA, Ferguson EC, Oldham SAA (2007) Lines and stripes: where did they go?-From conventional radiography to CT. Radiographics 27 (1): 33-48.

4. Sohoni CA (2013) Thickened and bulging left Paratracheal stripe: Is it always abnormal? Arch Med Health Sci 1:195-196. 